\title{
Two-dimensional superconductivity at a Mott insulator/band insulator interface $\mathrm{LaTiO}_{3} / \mathrm{SrTiO}_{3}$
}

\author{
J. Biscaras', N. Bergeal', A. Kushwaha², T. Wolf', A. Rastogi'², R.C. Budhani ${ }^{2,3}$ \& J. Lesueur
}

Transition metal oxides show a great variety of quantum electronic behaviours where correlations often have an important role. The achievement of high-quality epitaxial interfaces involving such materials gives a unique opportunity to engineer artificial structures where new electronic orders take place. One of the most striking result in this area is the recent observation of a two-dimensional electron gas at the interface between a strongly correlated Mott insulator $\mathrm{LaTiO}_{3}$ and a band insulator $\mathrm{SrTiO}_{3}$. The mechanism responsible for such a behaviour is still under debate. In particular, the influence of the nature of the insulator has to be clarified. In this article, we show that despite the expected electronic correlations, $\mathrm{LaTiO}_{3} / \mathrm{SrTiO}_{3}$ heterostructures undergo a superconducting transition at a critical temperature $T_{c}$ onset $_{\sim} 300 \mathrm{mK}$. We have found that the superconducting electron gas is confined over a typical thickness of $12 \mathrm{~nm}$ and is located mostly on the $\mathrm{SrTiO}_{3}$ substrate.

\footnotetext{
${ }^{1}$ Laboratoire de Physique et d'Etude des Matériaux, UMR8213/CNRS, ESPCI ParisTech, 10 rue Vauquelin, Paris 75005, France. ${ }^{2}$ Condensed Matter, Low Dimensional Systems Laboratory, Department of Physics, Indian Institute of Technology Kanpur, Kanpur 208016, India. ${ }^{3}$ National Physical Laboratory, New Delhi 110012, India. Correspondence and requests for materials should be addressed to N.B. (email: nicolas.bergeal@espci.fr).
} 
$\mathrm{P}$ erovskites-based structures including transition metal oxides have attracted much attention in the past decades, with the discovery of high- $T_{c}$ superconductivity and colossal magnetoresistance ${ }^{1}$. More generally, these compounds exhibit various electronic orders, going from the canonical anti-ferromagnetic (AF) Mott insulator when the onsite repulsion is maximum because of strong electronic interactions, to Fermi liquid-like metals when carrier doping is such that screening prevents the system from localization. Depending on the cations and the doping level involved, charge, spin and orbital orders can appear in the ground state together with metallic and even superconducting phases. Transitions between these states can be tuned by temperature, magnetic or electric fields ${ }^{2}$. All these compounds can be seen as stacks of oxide layers where the charge neutrality is conserved in the unit cell (u.c.), but not necessarily in each layer. Therefore, the translation symmetry is locally broken at the interface and charge imbalance can develop. Like in band-gap engineering with semiconductors, it is possible to create artificial interface materials by growing thin layers of a transition metal oxide on top of another one. Recently, the observation of two-dimensional (2D) superconductivity ${ }^{3}$ and magnetic correlations ${ }^{4}$ at the interface between the two band insulators $\mathrm{LaAlO}_{3}$ and $\mathrm{SrTiO}_{3}$ has drawn a lot of attention. Another particularly interesting candidate is the homo-metallic structure $\mathrm{LaTiO}_{3} / \mathrm{SrTiO}_{3}$ that uses $\mathrm{TiO}_{2}$ plans as a building block ${ }^{5,6}$. Titanium is in the $3 d^{0}$ state in the $\mathrm{SrTiO}_{3}$ layer which is a band insulator of $3.2 \mathrm{eV}$ bandgap, whereas it is $3 d^{1}$ in the $\mathrm{LaTiO}_{3}$ one which is therefore an AF Mott insulator due to strong correlations ${ }^{7}$. Providing the interface layer is $\mathrm{TiO}_{2}$, an extra electron is left in the structure every 2 u.c. ${ }^{8,9}$. As shown by photoemission $^{10}$ and optical studies ${ }^{11}$, a two-dimensionnal electron gas (2-DEG) develops and extends a few u.c. beyond the interface.

Several theoretical approaches pointed out that an electronic reconstruction leads to an increase of the electronic density at the $\mathrm{LaTiO}_{3} / \mathrm{SrTiO}_{3}$ interface ${ }^{8,9,12,13}$. Okamoto and Millis ${ }^{8}$ proposed a phase diagram where different orbital and magnetic states occur as a function of the thickness of the $\mathrm{LaTiO}_{3}$ layer and the strength of the Mott-Hubbard parameter $U / t$ ( $U$ is the Coulomb onsite repulsion energy, and $t$ the hopping term between neighbour $\mathrm{Ti}$ sites). Fully polarized ferromagnetic metallic sub-bands are expected to form for thickness below 5 u.c. and $U / t \sim 8-10$. However, Kancharla and Dagotto, ${ }^{14}$ taking into account both local and long-range Coulomb interactions, showed that strong AF fluctuations reminiscent of the magnetic order of the bulk compound persist in the metallic phase. As suggested by Larson ${ }^{15}$ and Okamoto ${ }^{16}$, lattice relaxation at the interface strongly modifies the band configuration, and may enhance the electronic correlations in the 2-DEG ${ }^{12}$. In this context, it is clear that the $\mathrm{LaTiO}_{3} / \mathrm{SrTiO}_{3}$ interface layer appears to be a unique system to study the physics of a 2-DEG influenced by strong electronic correlations. In this study, we show that this heterostructure undergoes a superconducting transition and we are able to characterize the 2-DEG as extending mostly towards the $\mathrm{SrTiO}_{3}$ side of the interface.

\section{Results}

Low-temperature transport measurements. We have grown epitaxial layers of $\mathrm{LaTiO}_{3}$ using pulsed laser deposition (PLD) on single crystal substrates of $\mathrm{SrTiO}_{3}$ cut along (100) and (110) crystallographic directions. The details of the growth conditions and X-ray characterizations are given in Methods section and in Supplementary Figures S1 and S2. In this study, we focus mainly on two $\mathrm{LaTiO}_{3} /(100) \mathrm{SrTiO}_{3}$ heterostructures whose thickness of 40 and $60 \AA$ correspond to 10 and 15 u.c. respectively. The sheet resistance measured in a Van der Pauw geometry decreases with temperature, indicating a metallic behaviour of the interface (Fig. 1). At temperatures lower than $20 \mathrm{~K}$ the two samples exhibit an increase of resistance characteristic of weak localization in disordered 2D films. The heterostructures undergo a superconducting transition at $T_{c}^{\text {onset }} \approx 310 \mathrm{mK}$ for the 10 u.c. sample and at $T_{c}^{\text {onset }} \approx 260 \mathrm{mK}$ for the 15 u.c. sample (inset, Fig. 1). Thinner 5 u.c. (100) films and 20 to 100 u.c. thicker (100) films as well as (110) oriented films are not metallic at low temperature.

In Figure 2, we show the current-voltage characteristics of the $10 \mathrm{u}$. c. sample measured at different temperatures. At low temperature, the $I(\mathrm{~V})$ curves show a clear critical current $I_{\mathrm{c}}$ of $5 \mu \mathrm{A}$ corresponding to a critical current per unit width of $16.7 \mu \mathrm{A} \mathrm{cm}^{-1}$. For current much higher than $I_{c}$, the $I(\mathrm{~V})$ curves merge together on a linear Ohmic law with a resistance corresponding to the normal resistance. In the case of the 15 u.c. sample, the critical current per unit width is found to be $14 \mu \mathrm{A} \mathrm{cm}^{-1}$. Figure $3 \mathrm{a}$ shows the sheet resistance of the $10 \mathrm{u}$.c sample as a function of temperature measured for different values of a magnetic field applied perpendicularly to the sample. The magnetic field induces a transition from a superconducting state to a nonsuperconducting one. The dependence of the critical field as a function of temperature is linear close to $T_{c}$, which is consistent with the

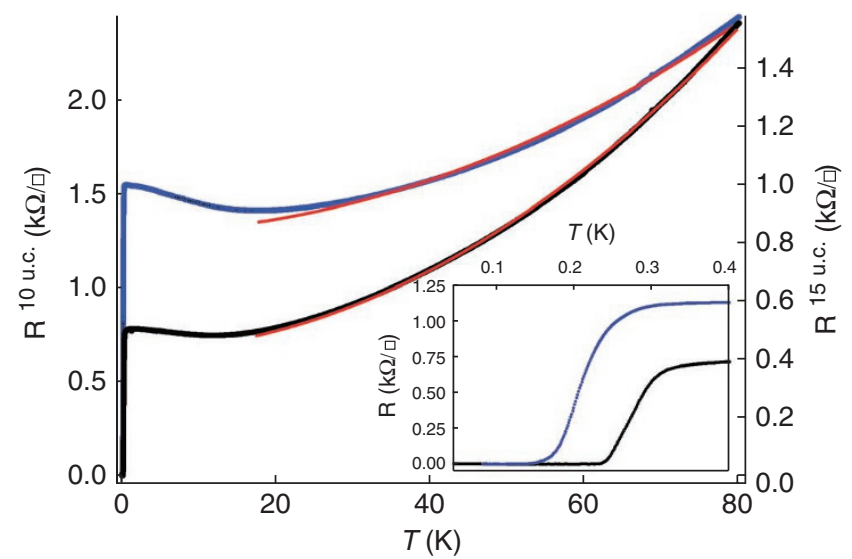

Figure 1 | Sheet resistance of the hererostructures. Sheet resistance a of the 10 u.c. (black dots, left axis) and 15 u.c. (blue dots, right axis) $\mathrm{LaTiO}_{3} / \mathrm{SrTiO}_{3}$ samples in an intermediate range of temperature. The red lines correspond to quadratic fits of the form $R(T)=A T^{2}+R_{0}$. (Inset) Sheet resistance as a function of temperature showing the superconducting transitions at $T_{c}^{\text {onset }}=310 \mathrm{mK}$ for the 10 u.c. (black dots) and $T_{c}$ onset $=260 \mathrm{mK}$ for the 15 u.c. (blue dots), where $T_{c}{ }_{c}^{\text {onset }}$ is defined by a $10 \%$ drop of the resistance.

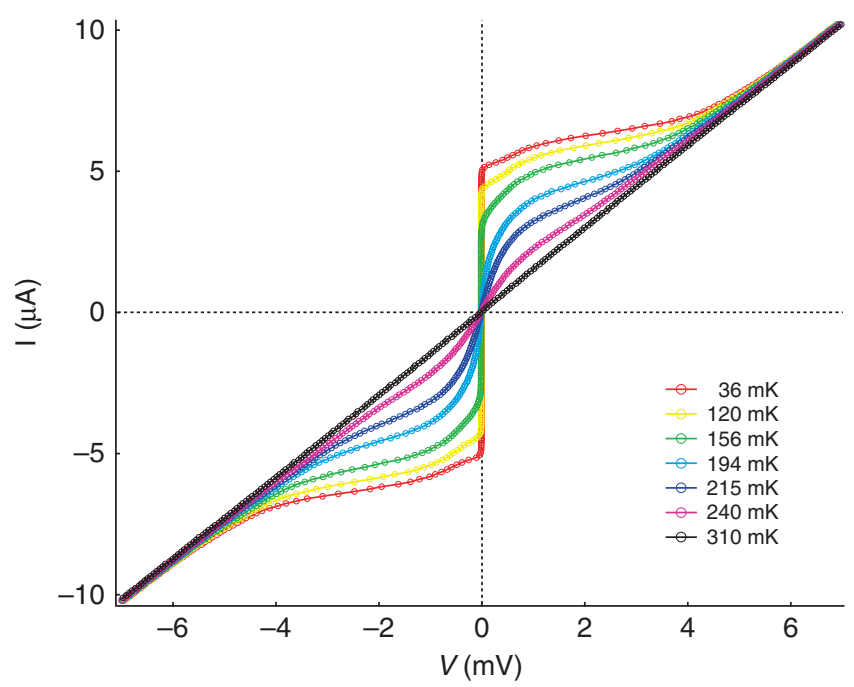

Figure 2 | Current-voltage characteristics of the 10 u.c. sample. The critical current at low temperature is $5 \mu \mathrm{A}$ corresponding to a critical current per unit width of $16.7 \mu \mathrm{Acm}^{-1}$. 

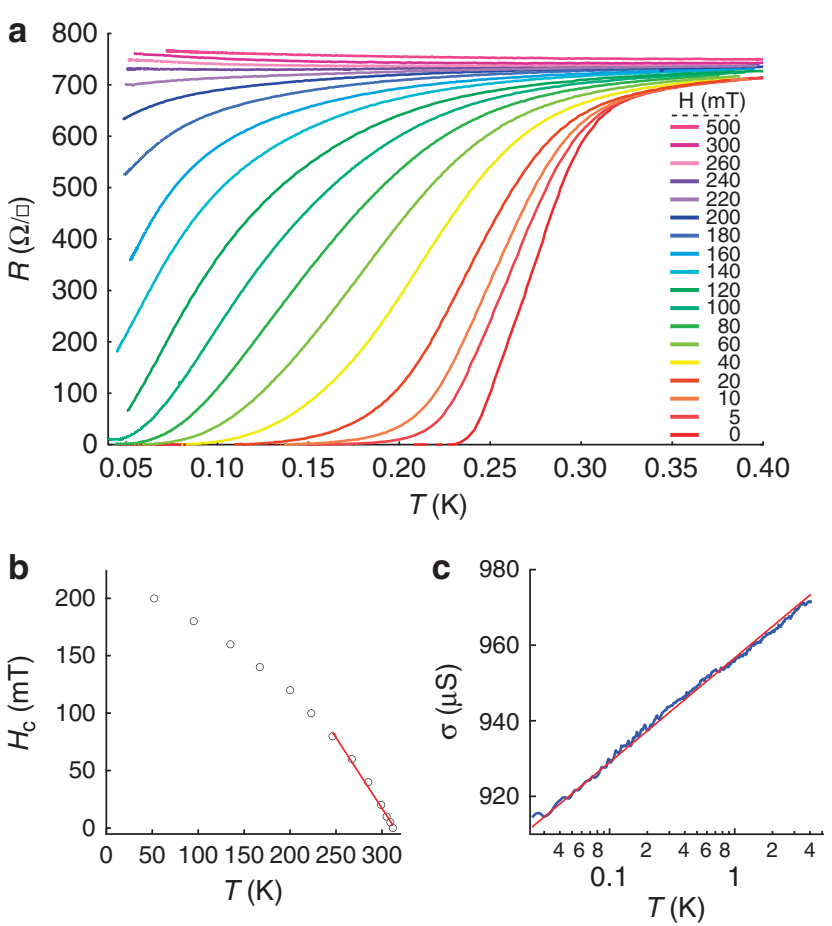

Figure 3 | Sheet resistance of the hererostructures under magnetic

field. (a) Sheet resistance of the 10 u.c. sample as a function of temperature for different values of the perpendicular magnetic field. (b) Temperature dependence of the perpendicular critical field, defined as the magnetic field that suppresses $90 \%$ of the resistance drop. The red line indicates the linear dependence of the critical field with temperature close to $T_{c}$. (c) Conductivity of the 15 u.c. sample as a function of temperature for a perpendicular magnetic field corresponding to the critical field. The red line corresponds to the expression $\sigma_{2 D}(T)=\sigma_{0}+\left(p e^{2} / \pi h\right) \ln \left(T / T_{0}\right)$ with $p=0.97$ indicating that phase coherence is limited by electron-electron scattering $(p=1)^{19}$.

form $H(T)=\Phi_{0} / 2 \pi \xi_{\|}^{2}(T)$ taking into account a Landau-Ginsburg in-plane coherence length $\xi_{\|} \propto\left(T_{c}-T\right)^{-1 / 2}$ (Fig. 3b). The critical field extrapolated at $T=0$ is $H_{\perp}^{c} \approx 220 \mathrm{mT}$ for the 10 u.c. sample and $H_{\perp}^{c} \approx 210 \mathrm{mT}$ for the 15 u.c. sample (see Supplementary Fig. S3). At $T=0$, we found $\xi^{10 u . c .}(T=0) \approx 38 \mathrm{~nm}$ and $\xi^{15 \text { u.c. }}(T=0) \approx 42 \mathrm{~nm}$. Measurements performed in a parallel magnetic field geometry give $H_{\|}^{c}=\sqrt{3} \Phi_{0} / \pi d \xi_{\|}(T=0) \approx 2.15 \mathrm{~T}$ for the 10 u.c sample and $H_{\|}^{c}=2.2 \mathrm{~T}$ for the 15 u.c sample. We thus extract the thickness of the 2D superconducting electron gas $d^{15 u . c} \approx 12 \mathrm{~nm}$ and $d^{15 u . c .} \approx 13.5 \mathrm{~nm}$. Note that this is an upper bound given the precision of the sample alignment in the parallel magnetic field. These values are close to the ones reported in $\mathrm{LaAlO}_{3} / \mathrm{SrTiO}_{3}$ heterostructures ${ }^{17,18}$. In disordered electronic system, weak localization produces a decrease of conductivity that can be experimentally revealed by varying the temperature. In the particular case of a $2 \mathrm{D}$ system, the conductivity takes the remarkable logarithmic dependence with temperature $\sigma_{2 D}(T)=\sigma_{0}+\left(p e^{2} / \pi h\right) \ln \left(T / T_{0}\right)$, where $p$ depends on the process that limits the phase coherence; $p=3$ for electronphonon scattering and $p=1$ for electron-electron scattering in the dirty limit ${ }^{19}$. Such logarithmic temperature dependence is observed on our samples (see Fig. 3c), thus confirming the 2D nature of the electron gas. The fit gives $p=0.97 \pm 0.05$ showing that the phase coherence is limited mainly by electron-electron scattering.

Hall effect measurements. To investigate the density and mobility of charge carriers, we performed Hall measurements at low temperature (Fig. 4). The experiment confirms that the sign of the hall coefficient $R_{H}=V_{H} / I B$ is negative for both samples, indicating that

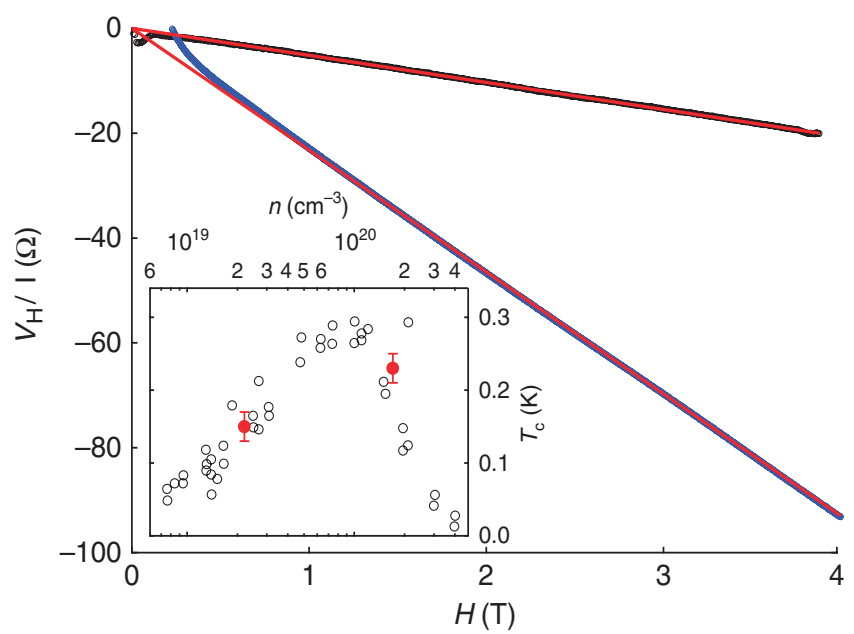

Figure 4 | Hall effect. Hall voltage $V_{H}$ divided by the current I as a function of magnetic field for the 10 u. c. (black dots) and 15 u.c. (blue dots) $\mathrm{LaTiO}_{3} / \mathrm{SrTiO}_{3}$ samples, measured at $100 \mathrm{mK}$. Red solid lines correspond to linear fits. (Inset) $T_{\mathrm{c}}$ as a function of doping for $\mathrm{SrTiO}_{3}$ single crystals taken from reference ${ }^{27}$ (black open circles). The two red dots correspond to $\mathrm{LaTiO}_{3} / \mathrm{SrTiO}_{3}$ samples taking the thickness $d^{10 \text { u.c. }}=12 \mathrm{~nm}, d^{15 \text { u.c. }}=13.5 \mathrm{~nm}$. Here, we have defined $T_{c}$ as the temperature at which the resistance reaches zero as this definition is more appropriate for comparison with the magnetic definition of the $T_{c}$ used in reference ${ }^{27}$. Errors bars represent the uncertainty in the comparison of the two definitions of $T_{c}$.

electron-like charge carriers dominate the transport. The sheet carrier density $n_{S}=1 / e R_{H}$ was found to be $2 \times 10^{14} \mathrm{~cm}^{2}$ for the 10 u.c. sample and $2.7 \times 10^{13} \mathrm{~cm}^{2}$ for the 15 u.c. one. Taking the sheet resistance measured previously, we obtained a Hall mobility $\mu=1 / e R_{s} n_{s}$ of $52 \mathrm{~cm}^{2} \mathrm{~V}^{-1} \mathrm{~s}^{-1}$ for the 10 u.c. sample and $210 \mathrm{~cm}^{2} \mathrm{~V}^{-1} \mathrm{~s}^{-1}$ for the 15 u.c. one. In an ideal picture, the interface between $\mathrm{SrTiO}_{3}$ and $\mathrm{LaTiO}_{3}$ can be observed as a Ti ions network, in the $4^{+}$state in $\mathrm{SrTiO}_{3}$ and in the $3^{+}$one in $\mathrm{LaTiO}_{3}$. Therefore, one electron is left every two cells on average at the interface, which corresponds to an areal density of approximately $3 \times 10^{14} \mathrm{~cm}^{-2}(\mathrm{refs} 8,9)$. This is approximatively the electron density measured through Hall effect in the 10 u.c. sample $\left(2 \times 10^{14} \mathrm{~cm}^{-2}\right)$, consistent with the value observed in $\mathrm{LaTiO}_{3} / \mathrm{SrTiO}_{3}$ superlattices ${ }^{6}$ by measuring the number of $T^{3+}$ in the vicinity of the interface. Optical studies confirm that free carriers with densities of approximately $3 \times 10^{14} \mathrm{~cm}^{-2}$ do exist in similar superlattices, with a typical mobility of $35 \mathrm{~cm}^{2} \mathrm{~V}^{-1} \mathrm{~s}^{-1}$ and an effective mass $m^{*} \simeq 2 m_{\mathrm{e}}{ }^{11}$. The mobility that we measured on the 10 u.c. sample $\left(52 \mathrm{~cm}^{2} \mathrm{~V}^{-1} \mathrm{~s}^{-1}\right)$ is close to this value, which supports an effective mass close to $2 m_{\mathrm{e}}$. The 15 u.c. has a lower sheet density of $2.7 \times 10^{13} \mathrm{~cm}^{-2}$ and a $T_{c}^{\text {onset }}$ of only $260 \mathrm{mK}$.

\section{Discussion}

It is known that $\mathrm{LaTiO}_{3}$ itself can be oxygen ${ }^{20-21}$ or $\mathrm{Sr}$ doped ${ }^{23}$, and thus becomes metallic. The key question is therefore: does superconductivity take place within a doped Mott insulator layer, namely oxygen- or Sr-doped $\mathrm{LaTiO}_{3}$, or within a 2-DEG formed at the $\mathrm{LaTiO}_{3} / \mathrm{SrTiO}_{3}$ interface, which extends mostly within the band insulator $\mathrm{SrTiO}_{3}$ (ref. 8)? And conversely, do the electronic correlations, which are known to be strong in the former case and moderate in the latter one ${ }^{16}$, have a role in that context? The recent works on $\mathrm{LaTiO}_{3} / \mathrm{SrTiO}_{3}$ superlattices ${ }^{6,11,24}$ clearly indicate that under proper growth conditions, the interface is abrupt, with no sizable Sr diffusion for deposition temperatures below $1,000^{\circ} \mathrm{C}^{10}$ Optical spectroscopy confirms that the carrier properties in superlattices are different from the ones in $\mathrm{La}_{1-x} \mathrm{Sr}_{x} \mathrm{TiO}_{3}$ compounds ${ }^{11}$. Moreover, the low-temperature transport properties are different 


\begin{tabular}{|c|c|}
\hline \multicolumn{2}{|c|}{$\begin{array}{l}\text { Table } 1 \text { | Comparison of } A \text { parameters (given in } \Omega \mathrm{cm} / K^{2} \text { ) } \\
\text { measured in the } 10 \text { u.c. and } 15 \mathrm{u} . \mathrm{c} \text {. } \mathrm{LaTiO}_{3} / \mathrm{SrTiO}_{3} \text { samples } \\
\text { (taking the thickness } d^{10 u . c .}=12 \mathrm{~nm}, d^{15 u . c .}=13.5 \mathrm{~nm} \text { ), with } \\
\text { data obtained from literature on thin films and crystals for } \\
\text { similar doping. }\end{array}$} \\
\hline Compound & $\mathrm{A}\left(\Omega \mathrm{cm} / \mathrm{K}^{2}\right)$ \\
\hline $\begin{array}{l}\text { Ox. } \mathrm{LaTiO}_{3}^{*} \\
\text { Sr. } \mathrm{LaTiO}_{3} \dagger \\
\mathrm{LaTiO}_{3}(10 \text { u.c. }) / \mathrm{SrTiO}_{3} \\
\text { LaTiO } \\
\text { La. } \mathrm{STO}_{\mathbf{t}}(15 \text { u.c. }) / \mathrm{SrTiO}_{3} \\
\text { La. STO }\end{array}$ & $\begin{array}{l}8 \times 10^{-9} \\
2 \times 10^{-9} \\
3.6 \times 10^{-7} \\
1.3 \times 10^{-7} \\
5 \times 10^{-7} \\
3 \times 10^{-8}\end{array}$ \\
\hline 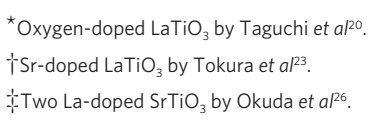 & \\
\hline
\end{tabular}

in conducting doped $\mathrm{LaTiO}_{3}$ and doped $\mathrm{SrTiO}_{3}$. In both cases, electron-electron collisions dominate the scattering events according to the Fermi liquid picture. Figure 1 shows that, in an intermediate regime temperature, the temperature dependence of the resistance is well fitted by a quadratic law $R(T)=A T^{2}+R_{0}$ where the coefficient $A$ depends on the Landau parameters, and therefore on the carrier density and the effective mass $\mathrm{m}^{\star 25}$. We obtained $A=0.27 \Omega / \square K^{2}$ for the 10 u.c. sample and $A=0.11 \Omega / \square K^{2}$ for the 15 u.c. sample. In Table 1, we summarize the different values of $A$ found in the literature for doped $\mathrm{LaTiO}_{3}$ and doped $\mathrm{SrTiO}_{3}$ thin films and crystals and compare them to the values extracted from our experiment. The largest values of $A$ reported in doped $\mathrm{LaTiO}_{3}$ are on the order of $1 \times 10^{-9} \Omega \mathrm{cm} / K^{2}$ before the system becomes insulating at low temperature $(\partial R / \partial T<0$ for $T<100 \mathrm{~K})$, whereas it is two orders of magnitude higher for doped $\mathrm{SrTiO}_{3}$. From the comparison, we see that the $\mathrm{LaTiO}_{3} / \mathrm{SrTiO}_{3}$ interface layer behave more like doped $\mathrm{SrTiO}_{3}$ than doped $\mathrm{LaTiO}_{3}$. These observations are consistent with an electronic reconstruction of the $\mathrm{LaTiO}_{3} / \mathrm{SrTiO}_{3}$ interface, leading to the formation of a few unit cells thick 2-DEG in the $\mathrm{SrTiO}_{3}$ that projects mostly towards the $\mathrm{SrTiO}_{3}$ substrate and makes it conducting layer ${ }^{8,15,16}$. As shown in Figure 4 inset, our data are consistent with the dependence of $T_{c}$ with the carrier density of doped $\mathrm{SrTiO}_{3}$ reported in the literature ${ }^{27}$.

In summary, we have measured the electronic transport properties of $\mathrm{LaTiO}_{3} / \mathrm{SrTiO}_{3}$ heterostructures. The samples show a metallic behaviour and a superconducting transition is observed at low temperature. Our analysis shows that a 2-DEG is formed at the interface which is located mostly on the $\mathrm{SrTiO}_{3}$ substrate, in agreement with the electronic reconstruction scenario ${ }^{8}$. This discovery opens the possibility to study the interplay between superconductivity and different electronic orders predicted to take place with ultra-thin $\mathrm{LaTiO}_{3}$ films on $\mathrm{SrTiO}_{3}$. According to our results in terms of carrier density, mobility and gas thickness, it should be possible to modulate significantly the behaviour of the 2-DEG by adjusting the number of charge carriers with an electrostatic gate.

\footnotetext{
Methods

Growth of $\mathrm{LaTiO}_{3} / \mathrm{SrTiO}_{3}$ heterostructures. We have grown epitaxial layers of $\mathrm{LaTiO}_{3}$ using excimer laser-based PLD on commercially available (Crystal) single-crystal substrates of $\mathrm{SrTiO}_{3}$ cut along (100) and (110) crystallographic directions. Although the (100) subtrates were given a buffered $\mathrm{HF}$ treatment to expose $\mathrm{TiO}_{2}$-terminated surface, the (110) plane has $\mathrm{Sr}$, Ti and oxygen ions on one plane and hence HF treatment is irrelevant in this case. The substrates were glued to the heater block of the PLD system and heated in oxygen pressure of 200 mtorr in the temperature range of $850-950^{\circ} \mathrm{C}$ for $1 \mathrm{~h}$ to realize surface reconstruction. This process has been used routinely to grow epitaxial films and heterostructures of $\mathrm{YBa}_{2} \mathrm{Cu}_{3} \mathrm{O}_{6+x}$ and hole-doped manganites. The source of $\mathrm{LaTiO}_{3}$ is a stoichiometric sintered target of $22 \mathrm{~mm}$ in diameter that was
}

ablated in oxygen partial pressure of $1 \times 10^{-4}$ torr with energy fluence of $\sim 1 \mathrm{Jcm}^{-2}$ per pulse at a repetition rate of $3 \mathrm{~Hz}$ to realize a gowth rate of $0.12 \AA^{-1}$.

$\mathrm{X}$-ray characterizations. The X-ray diffraction pattern of $\mathrm{LaTiO}_{3}$ films deposited on (100) $\mathrm{SrTiO}_{3}$ substrate is shown in Supplementary Figure S1. After subtracting the contribution of the substrate, the lattice parameter of the film is found to be $3.956 \AA$ (inset), in good agreement with previous studies ${ }^{28}$ and close to the one reported in bulk $\mathrm{LaTiO}_{3}(3.928 \AA)^{29}$. The X-ray diffraction pattern $(\theta-2 \theta$ scan $)$ around $32^{\circ}$ of $\mathrm{LaTiO}_{3}$ films deposited on $\mathrm{SrTiO}_{3}(110)$ is shown in panel a of Supplementary Figure S2. The (110) peak of the film is observed at $2 \theta=32.193^{\circ}$, close to the (110) peak of the substrate, which corresponds to a $\mathrm{LaTiO}_{3}$ lattice parameter of $3.928 \AA$. As shown in panel b of Supplementary Figure S2, the typical width of the rocking curve of the (110) peak is about $0.1^{\circ}$ indicating a very good out-of-plane orientation of the layers.

\section{References}

1. Dagotto, E. Complexity in strongly correlated electronic systems. Science 309, 257 (2005).

2. Imada, M., Fujimori, A. \& Tokura, Y. Metal-insulator transitions. Rev. Mod. Phys. 70, 1039-1263 (1998).

3. Reyren, N. et al. Superconducting interfaces between insulating oxides. Science 317, 1196-1199 (2007).

4. Brinkman, et al. Magnetic effects at the interface between non-magnetic oxides. Nat. Mater 6, 493-496 (2007).

5. Ohtomo, A. \& Hwang, H. Y. A high-mobility electron gas at the $\mathrm{LaAlO}_{3} /$ $\mathrm{SrTiO}_{3}$. Nature 427, 423-426 (2004).

6. Ohtomo, A., Muller, D. A., Grazul, J. L. \& Hwang, H. Y. Artificial chargemodulation in atomic-scale perovskite titanate superlattices. Nature 419, 378-380 (2002).

7. Tokura Fillingness dependence of electronic-structures in strongly correlated electron-systems-titanates and vanadates. J. Phys. Chem. Solids 53, 1619-1625 (1992).

8. Okamoto, S. \& Millis, A. J. Electronic reconstruction at an interface between a Mott insulator and a band insulator. Nature 428, 630-633 (2004).

9. Okamoto, S. \& Millis, A. J. Spatial inhomogeneity and strong correlation physics: a dynamical mean-field study of a model Mott-insulator-bandinsulator heterostructure. Phys. Rev. B 70, 241104 (2004).

10. Takizawa, M. Photoemission from buried interfaces in $\mathrm{SrTiO}_{3} / \mathrm{LaTiO}_{3} /$ superlattices. Phys. Rev. Lett. 97, 057601 (2006).

11. Seo, S. S. et al. Optical study of the free-carrier response of $\mathrm{LaTiO}_{3} / \mathrm{SrTiO}_{3}$ superlattices. Phys. Rev. Lett. 99, 266801 (2007).

12. Ishida, H. \& Liebsch, A. Origin of metallicity of $\mathrm{LaTiO}_{3} / \mathrm{SrTiO}_{3}$ heterostructures. Phys. Rev. 77, 115350 (2008).

13. Popovic, Z., Satpathy, S. \& Martin, R. M. Origin of the two-dimensional electron gas carrier density at the on $\mathrm{SrTiO}_{3}$ interface. Phys. Rev. Lett. 101, 256801 (2008).

14. Kancharla, S. S. \& Dagotto, E. Metallic interface at the boundary between band and Mott insulators. Phys. Rev. B 74, 195427 (2006).

15. Larson, P., Popović, Z. \& Satpathy, S. Lattice relaxation effects on the interface electron states in the perovskite oxide: $\mathrm{LaTiO}_{3}$ monolayer embedded in $\mathrm{SrTiO}_{3}$. Phys. Rev. B 77, 245122 (2008).

16. Okamoto, S., Millis, A. J. \& Spaldin, N. A. Lattice relaxation in oxide heterostructures: $\mathrm{LaTiO}_{3} / \mathrm{SrTiO}_{3}$ superlattices. Phys. Rev. Lett. 97, 056802 (2006).

17. Basletic, M. Mapping the spatial distribution of charge carriers in $\mathrm{LaTiO}_{3}$ / $\mathrm{SrTiO}_{3}$. Nat. Mater 7, 621-625 (2008).

18. Copie, O. et al. Towards two-dimensional metallic behavior at $\mathrm{LaTiO}_{3} / \mathrm{SrTiO}_{3}$ interfaces. Phys. Rev. Lett. 102, 216804 (2009).

19. Lee, P. A. \& Ramakrishnan, T. V. Disordered electronic systems. Rev. Mod. Phys. 57, 287-3317 (1985).

20. Taguchi, Y. et al. Critical behavior in $\mathrm{LaTiO}_{3+\delta / 2}$ in the vicinity of antiferromagnetic instability. Phys. Rev. B 59, 7917-7924 (1999).

21. Wang, F., Li, J., Wang, P., Zhu, X. \& Zhang, M. Effect of oxygen content on the transport properties of $\mathrm{LaTiO}_{3+\delta / 2}$ thin films. J. Phys. Condens. Matter 18, 5835-5847 (2006).

22. Gariglio, S., Seo, J. W., Fompeyrine, J., Locquet, J. -P. \& Triscone, J. -M. Transport properties in doped Mott insulator epitaxial $\mathrm{La}_{1-y} \mathrm{TiO}_{3+} \delta$ thin films. Phys. Rev. B 63, 161103 (2001).

23. Tokura, Y., Taguchi, Y., Okada, Y., Fujishima, Y., Arima, T., Kumagai, K. \& Iye, Y. Filling dependence of electronic properties on the verge of metal-Mottinsulator transition in $\mathrm{Sr}_{1-x} \mathrm{La}_{\mathrm{x}} \mathrm{TiO}_{3}$. Phys. Rev. Lett. 70, 2126-2129 (1993).

24. Shibuya, K., Ohnishi, T., Kawasaki, M., Koinuma, H. \& Lippmaa, M. Metallic $\mathrm{LaTiO}_{3} / \mathrm{SrTiO}_{3}$ superlattice films on the $\mathrm{SrTiO}_{3}$. Jpn. J. Appl. Phys. 43, L1178L1180 (2004)

25. Nozières, P. \& Pines, D. The Theory of Quantum Liquids (Perseus Books, 1999)

26. Okuda, T., Nakanishi, K., Miyasaka, S. \& Tokura, Y. Large thermoelectric response of metallic perovskites: $\mathrm{Sr}_{1-x} \mathrm{La}_{\mathrm{x}} \mathrm{TiO}_{3}$. Phys. Rev. B 63, 113104 (2001).

27. Koonce, C. S., Cohen, M. L., Schooley, J. F., Hosler, W. R. \& Pfeiffer, E. R. Superconducting transition temperatures of semiconducting $\mathrm{SrTiO}_{3}$. Phys. Rev. 163, 380-390 (1967). 
28. Havelia, S., Balasubramaniam, K. R., Spurgeon, S., Cormack, F. \& Salvador, P. A. Growth of $\mathrm{La}(2) \mathrm{Ti}(2) \mathrm{O}(7)$ and $\mathrm{LaTiO}_{3}$ thin films using pulsed laser deposition. J. Crystal Growth 310, 1985-1990 (2008).

29. Kestigian, M. \& Ward, R. The preparation of lanthanum titanium oxide, $\mathrm{LaTiO}_{3}$. J. Am. Chem. Soc. 76, 6027 (1954).

\section{Acknowledgments}

We acknowledge L. Benfatto, M. Grilli, S. Caprara, C. Castellani, C. Di Castro for useful discussions and L. Dumoulin for technical support. This work was supported by the Région Ile-de-France in the framework of CNano IdF and Sesame program. CNano IdF is the nanoscience competence centre of Paris Region, supported by CNRS, CEA, MESR and Region Ile-de-France. Research in India was funded by the Department of Information Technology, Government of India.

\section{Author contributions}

A.K., A.R. and R.C.B. prepared the samples and the X-ray analysis. J.B. and N.B. performed the measurements, assisted by T.W. J.B., N.B., R.C.B. and J.L. carried out the analysis of the results and written the article.

\section{Additional information}

Supplementary Information accompanies this paper on http://www.nature.com/ naturecommunications

Competing financial interests: The authors declare no competing financial interests.

Reprints and permission information is available online at http://npg.nature.com/ reprintsandpermissions/

How to cite this article: Biscaras, J. et al. Two-dimensional superconductivity at a Mott insulator/band insulator interface $\mathrm{LaTiO}_{3} / \mathrm{SrTiO}_{3}$. Nat. Commun. 1:89 doi: $10.1038 /$ ncomms1084 (2010). 